\title{
Olivopontocerebellar atrophy-deafness syndrome
}

INSERM

\section{Source}

INSERM. (1999). Orphanet: an online rare disease and orphan drug data base.

Olivopontocerebellar atrophy-deafness syndrome. ORPHA:2732

Olivopontocerebellar atrophy-deafness syndrome is characterised by infancy-onset olivopontocerebellar atrophy, sensorineural deafness and speech impairment. It has been described in less than 15 children. Most cases were sporadic, but autosomal recessive inheritance was suggested in three cases. 